ORIGINAL ARTICLE

\title{
Weight gain in patients with Obstructive Sleep Apnea (OSA) on Positive Airway Pressure (PAP) therapy - Myth or Reality?
}

\author{
Anup Bansal, Pavan Yadav, Nagarajan Ramakrishnan
}

Nithra Institute of Sleep Sciences, Chennai, India

DOI No: 10.5958/0974-0155.2014.01095.X

Indian J Sleep Med 2013; 8.4, 164-166

\begin{abstract}
Obesity is recognized as one of the predominant risk factor for Obstructive Sleep Apnea (OSA). Even moderate reduction in weight and body mass index (BMI) could reduce the severity of OSA and is recommended as part of comprehensive therapeutic approach in patients who are prescribed Positive Airway Pressure (PAP) therapy. However, this remains a challenge for most patients and some may even report weight gain. In this article, we report a case history highlighting weight gain while on PAP therapy and review the literature on this phenomenon.
\end{abstract}

Keywords: Obstructive Sleep apnea, Continuous positive airway pressure, Leptin, Body mass index.

\section{Introduction}

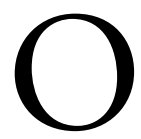

bstructive Sleep Apnea (OSA) and body weight are closely linked in several ways. Obesity has been the most talked about risk factor for OSA and it has been shown that weight loss is an important factor in comprehensive management of these patients ${ }^{1}$. Hormones like leptin, ghrelin adiponectin and resistin play an important role in regulation of body weight ${ }^{2}$. These hormones are directly linked to insulin resistance and obesity. Although reduction in BMI is expected in patients on CPAP therapy, literature reveals that some

Address for correspondence Nagarajan Ramakrishnan $A B$ (Int Med)

$A B$ (Crit Care), AB (Sleep Med) MMM, FACP, FCCP, FCCM, FISDA, FICCM

Senior Consultant \& Director Nithra Institute of Sleep Sciences

Door \# 29 (Plot \# 1997), J Block, 13 ${ }^{\text {th }}$ Main Road Annanagar, Chennai 600040

Ph: +91 444350 2252; Email: ram@nithra.com individuals gain weight possibly because of changes in insulin sensitivity or adipokines levels. Reduction in energy expenditure may also be a possible mechanism of weight gain in some OSA patients. It remains controversial whether CPAP therapy leading to improvement in hypoxia related sympathetic activation is the main reason for weight changes in OSA patients.

We encountered a patient who reported significant weight gain while on PAP therapy and was quoting this as a reason for poor compliance. A literature search using PUBMED does not reveal case reports from India highlighting this issue. Interestingly, Viswanathan et al have demonstrated that subjects with Type 2 Diabetes may have OSA even at lower BMI Values ${ }^{3}$. It would be of importance for clinicians to be sensitive to this issue as weight gain in such non-obese patients while on treatment with PAP may have implications on their management of diabetes and related comorbidities. This article provides a brief description of the case and a discussion on existing literature on weight gain while on PAP therapy. 


\section{Case Report}

Fifty nine year old obese gentleman with BMI of 36.5 and co-morbidities including hypertension, and chronic myeloid leukemia was evaluated for snoring and excessive daytimes sleepiness. On the basis of polysomnography study done in a sleep center at United States, he was diagnosed with severe OSA with Apnea-Hypopnea Index (AHI) of 68.3 and significant oxygen desaturations (nadir: $80 \%$ ). Arterial Blood Gas analysis done on room air at rest did not reveal any significant hypoxemia or hypercarbia. He was prescribed nocturnal Continuous Positive Airway Pressure (CPAP) therapy at home. After 3 months of CPAP therapy with documented good compliance, he did not observe significant improvement in daytime hypersomnolence. A brief trial of Modafinil was of some benefit but patient expressed unhappiness over the lack of improvement. A repeat polysomnogram at this time suggested a clinical diagnosis of complex sleep disordered breathing and he was switched over to Adaptive Servo bi-level ventilation. While noting an improvement in his daytime symptoms on the modified regimen, he now complained of weight gain over $6 \mathrm{~kg}$ since initiation of CPAP therapy. He was sleeping at least 7 hours and waking up feeling refreshed and remained active during the day. He had also initiated an exercise regimen for weight loss. It was discussed that his oral medication for chronic myeloid leukemia could be a contributing factor for the weight change. At this time patient admitted that he had 'tried without PAP for almost a month' when he noticed that he was losing weight. However, when he resumed PAP therapy, he was frustrated that he regained weight making it more likely to be the primary contributing factor for weight gain. He wanted an explanation on whether the therapy (PAP) was contributing to the weight gain.

\section{Discussion}

OSA is characterized by signs of disturbed sleep (snoring, choking and restlessness) and daytime sleepiness. The strongest risk factor for OSA has been obesity; as is evidenced by the $40 \%$ prevalence of OSA in the obese ${ }^{4}$. Central obesity due to excessive accumulation of brown fat is an important stimulus for satiety hormone (Leptin) which regulates energy intake and expenditure. Most OSA patients have higher leptin levels than comparable cohorts with similar BMI who do not have sleep apnea5 Increase in leptin resistance in turn leads to increase caloric intake and makes weight management difficult for OSA patients. Earlier studies and reports suggested that CPAP was the most common and effective way of reducing weight in obese OSA patients. Contrary to the popular belief that CPAP therapy alone can lead to weight loss there have been several studies proving it ineffective and in the contrary leading to weight gain. Redenius et $\mathrm{al}^{6}$ found that some OSA patients initially lost weight after initiation of CPAP therapy but subsequently most of them gained weight. These significant BMI changes were particularly more common in non obese CPAP users. In this study they have not monitored the leptin levels but previously Harsh et al have clearly demonstrated reduction in leptin levels following CPAP therapy in OSAS patients ${ }^{7}$. Decreasing leptin levels in turn stimulate appetite and more caloric intake which results in weight gain. Interestingly, they have also observed BMI changes were more profound in patients with great adherence to CPAP therapy.

It is also well established fact that obesity is related to ratio of energy intake to energy expenditure (EE) in all individuals even in OSA patient's also. Normally in OSA subjects, EE was significantly increased during sleep mainly during apnoeic episodes but this reduces with CPAP treatment ${ }^{8}$. This could be one of the mechanisms of weight gain, despite the fact that their daytime energy expenditure may not be significantly altered or even increased due to alertness levels and increased activity.

Some studies have showed that weight gain in OSA patient is seen only for a short period of time after starting CPAP treatment and not sustained in the long term. Retrospective study by Anas Balaa et al concluded that positive pressure therapy for OSA is associated with gain in body mass at one month but not after 6 months of treatment. The authors state the lack of understanding on the etiology but attribute it to an increase in vascular volume soon after initiation of PAP therapy ${ }^{9}$. Another retrospective study by Amarbil mattewal et al also observed a weight gain on CPAP at about 2 years after initiation, in a cohort of predominantly older males ${ }^{10}$.

In 2011, Jose M Garcia et al evaluated weight and metabolic effects of CPAP in obese obstructive sleep apnea patients. They have assayed insulin and appetite regulating hormones in all OSA patients before and after the initiation of CPAP therapy. Patients showed increased insulin resistance (IR) and decreased ghrelin levels while adipokines levels were remain unchanged. Interestingly, $40 \%$ of patients gained weight and it was 
directly correlated to changes in insulin sensitivity and ghrelin levels ${ }^{11}$.

Recently published study in THORAX in 2012 showed clear association between baseline ODI (oxygen desaturation index) and duration of desaturation below $90 \%$ on glucose/insulin homeostasis indices. They also concluded smaller improvement in glucose-insulin homeostasis leads to less reduction in fat mass and visceral adiposity after I year of CPAP therapy. So even with good CPAP compliance and lifestyle intervention program, metabolic effects and body weight reduction will be less in OSA patients. The researchers showed that OSA would attenuate the effects of a lifestyle intervention program in men with visceral obesity ${ }^{12}$.

\section{Conclusion}

It is a common misconception amongst patients and some clinicians that initiation of CPAP therapy will lead to weight loss or at least prevent weight gain. CPAP is well accepted to improve daytime functionality by abolishing or reducing nocturnal apneas and oxygen desaturations. However, this may not translate to sustained weight loss. Lifestyle modifications and close follow up of contributing factors are important aspects of comprehensive disease management programs for patients with OSA. Clinicians should reinforce the value of sustained weight loss but remain empathetic to patients who genuinely are unable to achieve weight loss and may even gain weight while being compliant with prescribed CPAP therapy.

\section{References}

1. Surendra K. Sharma, Swastik Agarwal, Deepak Damodaran, et al .CPAP for the metabolic syndrome in patients with obstructive sleep apnea. N Engl J Med. 2011 Dec 15; 365:2277-86.

2. Gami AS, Caples SM, Somers VK. Obesity and obstructive sleep apnea. Endocrinol Metab Clin North Am 2003; 32: 869-94.
3. Vijay Viswanathan, N Ramakrishnan, S Sunaina, et al .Subjects with Type 2 Diabetes may have Obstructive Sleep Apnea even at lower BMI values. Indian J Sleep Med 2012; 7.2: 45-47.

4. Chin K, Shimizu K, Nakamura T, et al. Changes in intraabdomi-nal visceral fat and serum leptin levels in patients with obstructive sleep apnea syndrome following nasal continuous positive airway pressure therapy. Circulation 1999; 100:706-12.

5. Cuhadaroglu C, Utkusavas A, Ozturk L, Salman S, Ece T: Effects of nasal CPAP treatment on insulin resistance, lipid profile, and plasma leptin in sleep apnea. Lung 2009, 187(2):75-81.

6. Rachel Redenius, Carli Murphy, Erin O'Neill, Majed AlHamwi, Sarah Nath Zallek. Does CPAP Lead to Change in BMI? J Clin Sleep Med. 2008 June 15; 4(3): 205-209.

7. Stenlof K, Grunstein R, Hedner J, Sjostrom L. Energy expendi-ture in obstructive sleep apnea: effects of treatment with continu-ous positive airway pressure. Am J Physiol 1996; 271:E1036-43.

8. Harsch IA, Konturek PC, Koebnick C, et al. Leptin and ghrelin levels in patients with obstructive sleep apnoea: effect of CPAP treatment. Eur Respir J 2003; 22:251-7.

9. Anas Balaa, Ahmed M. Abugiazya, Raymond Bourey. Early gain in body mass with continuous positive airway pressure therapy for obstructive apnea. Chest. 2009. 136(4_MeetingAbstracts):70S. Doi: Abstract / October 2009.

10. Amarbir Mattewal, Supriya Singh, Max Hirshkowitz, Amir Sharafkhaneh et al Effect of Continuous Positive Airway Pressure (CPAP) Therapy on Weight in Patients with Obstructive Sleep Apnea (OSA)-A Record Review Study. Chest 2011;140(4 MeetingAbstracts): 1070A doi:10.1378/chest. 1119750 .

11. Jose M Garcia, Hossein Sharafkhaneh, Max Hirshkowitz, Rania Elkhatib and Amir Sharafkhaneh. Weight and metabolic effects of cpap in obstructive sleep apnea patients with obesity. Respiratory Research 2011, 12:80.

12. Borel AL, Leblanc $X$, Almeras $N$, et al. Sleep apnoea attenuates the effects of a lifestyle intervention programme in men with visceral obesity. Thorax. 2012 Aug; 67(8):735 41. 10. 